\title{
Frames and Coorbit Theory on Homogeneous Spaces with a Special Guidance on the Sphere
}

\author{
Stephan Dahlke, Gabriele Steidl, and Gerd Teschke \\ Communicated by M. Holschneider
}

\begin{abstract}
The topic of this article is a generalization of the theory of coorbit spaces and related frame constructions to Banach spaces of functions or distributions over domains and manifolds. As a special case one obtains modulation spaces and Gabor frames on spheres. Group theoretical considerations allow first to introduce generalized wavelet transforms. These are then used to define coorbit spaces on homogeneous spaces, which consist of functions having their generalized wavelet transform in some weighted $L_{p}$ space. We also describe natural ways of discretizing those wavelet transforms, or equivalently to obtain atomic decompositions and Banach frames for the corresponding coorbit spaces. Based on these facts we treat aspects of nonlinear approximation and show how the new theory can be applied to the Gabor transform on spheres. For the $S^{1}$ we exhibit concrete examples of admissible Gabor atoms which are very closely related to uncertainty minimizing states.
\end{abstract}

\section{Introduction}

In this article, we study the problem of analyzing functions on the sphere by means of Gabor frames. To analyze a given signal, the first step is always to decompose it into suitable building blocks. These building blocks depend on the given situation, they may

\footnotetext{
Math Subject Classifications. 57S25, 42C15, 42C40, 46E15, 46E35.

Keywords and Phrases. Square integrable group representations, time-frequency analysis, atomic decompositions, (Banach) frames, homogeneous spaces, weighted coorbit spaces.

Acknowledgements and Notes. First author was partially supported by Deutsche Forschungsgemeinschaft, Grant Da 360/4-3, and by the EU Research Training Network Harmonic Analysis and Statistics for Image Processing; second author was partially supported by Deutsche Forschungsgemeinschaft, Grant We 2602/2-1; third author was partially supported by Deutsche Forschungsgemeinschaft, Grants Te 354/1-3, Te 354/3-1, Te 354/4-1, Te 354/5-1 and by the EU Research Training Network Harmonic Analysis and Statistics for Image Processing.
} 
consist of the elements of an (orthonormal) basis, of a frame, or even of the elements of huge dictionaries. At first sight, an orthonormal or more general a Riesz basis seems to be the most promising choice since then the representation of the signal is unique and usually very fast decomposition and reconstruction algorithms are available. However, in recent studies, it has turned out that the Riesz basis setting is very often not flexible enough. Indeed, when dealing with complicated domains or manifolds, such a suitable Riesz basis might not exist or it might suffer from serious problems such as a lack of numerical stability. Moreover, in other applications such as in Gabor analysis a Balian-Low theorem [15] prohibits the existence of a good basis.

One way to overcome these difficulties is to work with a weaker concept, i.e., to work with frames. Frames provide stable and usually redundant nonorthogonal expansions in a Hilbert space $\mathcal{H}$. In general, a countable set of elements $\left\{e_{i}\right\}_{i \in \mathbb{Z}}$ is called a frame if there exist constants $0<A_{1} \leq A_{2}<\infty$ such that

$$
A_{1}\|f\|_{\mathcal{H}}^{2} \leq \sum_{i \in \mathbb{Z}}\left|\left\langle f, e_{i}\right\rangle_{\mathcal{H}}\right|^{2} \leq A_{2}\|f\|_{\mathcal{H}}^{2} .
$$

Recent studies indeed indicate that frames on domains and manifolds are much easier to construct than a Riesz basis, see, e.g., [3, 4, 17]. In this overview article, we are especially interested in the construction of Gabor frames on a very prominent manifold, the sphere. The approach presented here has been introduced and discussed in its full generality in [3, $4,5]$. It is essentially based on square integrable group representations and generalizes the well-known coorbit space theory developed by Feichtinger and Gröchenig in a series of articles, e.g., $[8,9,10,11,14]$. The important Feichtinger-Gröchenig theory has the following advantages:

- The theory is universal in the following sense: Given a Hilbert space $\mathcal{H}$ and a square integrable representation of a group $G$, the whole abstract machinery can be applied.

- The approach provides us with natural families of smoothness spaces, the coorbit spaces. They are defined as the collection of all elements in the Hilbert space $\mathcal{H}$ for which the voice transform associated with the group representation has a certain decay. In many cases, e.g., for the affine group and the Weyl-Heisenberg group, these coorbit spaces coincide with classical smoothness spaces such as Besov and modulation spaces, respectively.

- The Feichtinger-Gröchenig theory does not only give rise to Hilbert frames in $\mathcal{H}$, but also to frames in scales of the associated coorbit spaces. Moreover, not only Hilbert spaces, but also Banach spaces can be handled. It is important to note that the theory provides us with universal construction methods, that is all the building blocks (e.g., the synthesis operators) are the same for the whole scale of spaces.

- The discretization process that produces the frame does not take place on the manifold (which might look ugly and complicated), but on the Lie group at hand (which is usually a more handy object), and is transported to the manifold by the group representation.

So far, the Feichtinger-Gröchenig theory is well-established for problems on the whole Euclidean plane. In view of the advantages stated above, it seems to be quite natural to apply this approach also to compact manifolds such as the spheres. However, then one is usually faced with the following problem: In many cases, there exist group representations, but they are not square integrable since the groups are 'too large' with respect to the manifold. 
Therefore it is necessary to make the group 'smaller' which can be performed by factoring out a suitable subgroup. Then one has to work with coset spaces. This might cause serious problems since usually the group structure gets lost. Nevertheless, in a small series of articles $[3,4,5]$ we have shown that a generalization of the Feichtinger-Gröchenig theory to representations of quotient spaces is indeed possible.

In this article, we present the basic steps of this construction. Special emphasis is laid on the application to the spheres. We show that by combining our approach with the local Fourier transform on the spheres as introduced by Torressani [18], one obtains generalized modulation spaces on the spheres and atomic decompositions and Banach frames for these scales of spaces. We recall the principle ideas without going into details, but nevertheless the proofs of the central Theorems 3 and 4 that provide us with atomic decompositions and Banach frames, respectively, are briefly sketched. We also discuss a new aspect of constructing atomic decompositions and Banach frames on spheres, namely uncertainty relations. Given a square integrable group representation, one obtains a set of self-adjoint differential operators by taking the derivatives of the representation at the identity element. Then any pair of these operators gives rise to 'spherical' Heisenberg uncertainty principles, and those vectors that minimize the uncertainty can be interpreted as canonical analyzing atoms.

This article is organized as follows. In Section 2, we briefly review the group theoretical background and introduce the generalized wavelet transform. In Section 3, we define associated coorbit spaces and state the basic correspondence principle. The main results are then given in Section 4. In this section we formulate conditions for the existence of atomic decompositions and Banach frames. After having established decomposition (and therewith approximation) principles, the next natural goal is to determine the quality of approximation. This is the topic of Section 5. As the special focus of this article, we consider in Section 6 the application of the general theory to the construction of local Gabor frames and associated modulation spaces on spheres. As a new aspect, we state related uncertainty principles for the local Fourier transform on the sphere $S^{1}$ and compute the corresponding minimizing states in Section 6.2. Since these minimizing states do not meet all the conditions of the proposed coorbit theory, we suggest a reasonable approximation.

\section{Square Integrable Representations Modulo Subgroups}

Let $G$ be a locally compact group with left Haar measure $v$ and let $\mathcal{U}$ be a strongly continuous, unitary representation of $G$ on a separable Hilbert space $\mathcal{H}$. We say that $\mathcal{U}$ is square integrable if there exists $\psi \in \mathcal{H} \backslash\{0\}$ such that

$$
\int_{G}\left|\langle\psi, \mathcal{U}(g) \psi\rangle_{\mathcal{H}}\right|^{2} d \nu(g)<\infty .
$$

For the classical integral transforms like the short time Fourier transform and the wavelet transform related to the reduced Weyl-Heisenberg-group and the affine group, respectively, the representations in question are in fact square-integrable. However, for integral transforms related to group representations on $L_{2}$-spaces on manifolds, for example, on the sphere, square integrability usually fails to hold. In other words, the corresponding group is too large.

A way to overcome this fact is to factor out a suitable closed subgroup $H$. In this way, we restrict the representation to a quotient $X:=G / H$ which always admits a strongly quasi-invariant measure. Since in general the representation is not directly defined on $X$ we need to introduce a section $\sigma: X \rightarrow G$ which assigns to each coset a point lying in it. In 
other words, if $\Pi: G \rightarrow X$ denotes the canonical projection then $\Pi \circ \sigma=$ Id. It is always possible to choose a measurable section or even a continuous one on some dense open subset of $X$. In many examples the section will be continuous. For technical reasons we assume further that $G$ and, hence, also $X=G / H$ is $\sigma$-compact. A unitary representation $\mathcal{U}$ of $G$ on $\mathcal{H}$ is called square-integrable modulo $(H, \sigma)$ if there exists a function $\psi \in \mathcal{H}$ such that the self-adjoint operator $A_{\sigma}: \mathcal{H} \rightarrow \mathcal{H}$ weakly defined by

$$
A_{\sigma} f:=\int_{X}\langle f, \mathcal{U}(\sigma(x)) \psi\rangle_{\mathcal{H}} \mathcal{U}(\sigma(x)) \psi d \mu(x),
$$

is bounded and has a bounded inverse $A_{\sigma}^{-1}$. The function $\psi$ is called admissible. If $A_{\sigma}$ is a multiple of the identity then $\psi$ is called strictly admissible. In this article, we restrict our attention to the strictly admissible setting, where $A_{\sigma}=$ Id. More general operators $A_{\sigma}$ were considered in [5].

The wavelet transform $V_{\psi}: \mathcal{H} \rightarrow L_{2}(X)$ is defined by

$$
V_{\psi} f(x):=\langle f, \mathcal{U}(\sigma(x)) \psi\rangle_{\mathcal{H}}, \quad x \in X .
$$

Note that the set $S_{\sigma}:=\{\mathcal{U}(\sigma(x)) \psi: x \in X\}$ is total in $\mathcal{H}$, i.e., $S_{\sigma}^{\perp}=\{0\}$. Based on the wavelet transform we introduce the Hermitian kernel

$$
R_{\psi}(x, y)=V_{\psi}(\mathcal{U}(\sigma(x)) \psi)(y)=\langle\mathcal{U}(\sigma(x)) \psi, \mathcal{U}(\sigma(y)) \psi\rangle_{\mathcal{H}} .
$$

Then $V_{\psi}$ gives rise to the following correspondence between $\mathcal{H}$ and the reproducing kernel Hilbert space

$$
\mathcal{M}_{2}:=\left\{F \in L_{2}(X):\left\langle F, R_{\psi}(x, \cdot)\right\rangle=F(x) \text { a.e. }\right\} .
$$

Theorem 1 (Correspondence between $\mathcal{H}$ and $\mathcal{M}_{2}$ ). Let $\mathcal{U}$ be a square integrable representation of $G \bmod (H, \sigma)$ with a strictly admissible function $\psi$. Suppose that $V_{\psi}$ and $R_{\psi}$ are defined by (2.1) and (2.2), respectively. Then $V_{\psi}$ is a bijection of $\mathcal{H}$ onto the reproducing kernel Hilbert space $\mathcal{M}_{2}$.

For a proof see, e.g., [1, Theorem 7.3.1]. Furthermore, $V_{\psi}$ is an isometry such that it can be inverted on its range by its adjoint $V_{\psi}^{*}$. For $f \in \mathcal{H}$, we have the reconstruction formula $f=V_{\psi}^{*} V_{\psi} f$.

\section{Weighted Coorbit Spaces on Homogeneous Spaces}

We want to extend our consideration from $L_{2}(X)$ to more general weighted $L_{p}$-spaces. For some positive, measurable weight function $v$ on $X$ and $1 \leq p \leq \infty$, let

$$
L_{p, v}(X):=\left\{f \text { measurable }: f v \in L_{p}(X)\right\}
$$

with $\|f\|_{L_{p, v}}:=\left(\int_{X}|f(x)|^{p} v(x)^{p} d \mu(x)\right)^{1 / p}, 1 \leq p<\infty$ and $\|f\|_{L_{\infty, v}}:=$ ess $\sup _{x \in X}|f(x)| v(x)$.

The spaces $\mathcal{H}_{1, w}$ and $\mathcal{H}_{1, w}^{\prime}$. First we need to provide a suitable large reservoir for the objects of our coorbit spaces. To this end, let $w$ be some weight function on $X$ satisfying $w(x) \geq 1$ for all $x \in X$. Throughout this article, we impose the fundamental condition

$$
\text { ess } \sup _{y \in X} \int_{X}\left|R_{\psi}(x, y)\right| \frac{w(x)}{w(y)} d \mu(x)<\infty .
$$


By the Generalized Young Inequality in Theorem A.1, this implies that $R_{\psi}(x, \cdot)=$ $V_{\psi}(\mathcal{U}(\sigma(x)) \psi)$ is in $L_{1, w}$ and that the map $F \mapsto\left\langle F, R_{\psi}(x, \cdot)\right\rangle$ is continuous on $L_{1, w}$. Then the linear space

$$
\mathcal{H}_{1, w}:=\left\{f \in \mathcal{H}: V_{\psi}(f) \in L_{1, w}(X)\right\}
$$

together with the norm $\|f\|_{\mathcal{H}_{1, w}}:=\left\|V_{\psi} f\right\|_{L_{1, w}}$ becomes a Banach space which is continuously embedded in $\mathcal{H}$. In particular, since $R_{\psi}(x, \cdot) \in L_{1, w}(X)$ we see that $U(\sigma(x)) \psi \in$ $\mathcal{H}_{1, w}$. Introducing the dual space $\mathcal{H}_{1, w}^{\prime}$ of $\mathcal{H}_{1, w}$ we have the continuous embeddings $\mathcal{H}_{1, w} \subset \mathcal{H} \subset \mathcal{H}_{1, w}^{\prime}$, where $\mathcal{H}_{1, w}$ is norm dense in $\mathcal{H}$ and $\mathcal{H}$ is weak-* dense in $\mathcal{H}_{1, w}^{\prime}$. In other words, $\left(\mathcal{H}_{1, w}, \mathcal{H}, \mathcal{H}_{1, w}^{\prime}\right)$ forms a Gelfand triple. Now the operator $V_{\psi}$ can be extended to an operator on $H_{1, w}^{\prime}$ by

$$
V_{\psi} f(x):=\langle f, \mathcal{U}(\sigma(x)) \psi\rangle_{H_{1, w}^{\prime} \times H_{1, w}} .
$$

By (3.1), we see that $V_{\psi}: \mathcal{H}_{1, w}^{\prime} \rightarrow L_{\infty, 1 / w}(X)$ is a continuous operator. Then an operator $\tilde{V}_{\psi}$ can be weakly defined on $L_{\infty, 1 / w}(X)$ by

$$
\left\langle\tilde{V}_{\psi} F, g\right\rangle_{\mathcal{H}_{1, w}^{\prime} \times \mathcal{H}_{1, w}}:=\left\langle F, V_{\psi} g\right\rangle \quad \text { for all } g \in H_{1, w} .
$$

It can be shown that $\tilde{V}_{\psi}$ is a bounded operator on $L_{\infty, 1 / w}(X)$ and we obtain for $F \in$ $L_{\infty, 1 / w}(X)$

$V_{\psi} \tilde{V}_{\psi} F(x)=\left\langle\tilde{V}_{\psi} F, \mathcal{U}(\sigma(x)) \psi\right\rangle_{\mathcal{H}_{1, w}^{\prime} \times \mathcal{H}_{1, w}}=\left\langle F, V_{\psi}(\mathcal{U}(\sigma(x)) \psi)\right\rangle=\left\langle F, R_{\psi}(x, \cdot)\right\rangle$

Weighted coorbit spaces. Now we introduce the weighted coorbit spaces. To this end, let $v$ be a positive measurable weight function on $X$. We impose the fundamental conditions

ess $\sup _{y \in X} \int_{X}\left|R_{\psi}(x, y)\right| \frac{v(x)}{v(y)} d \mu(x)<\infty$, ess $\sup _{x \in X} \int_{X}\left|R_{\psi}(x, y)\right| \frac{v(x)}{v(y)} d \mu(y)<\infty$.

By the Generalized Young Inequality in Theorem A.1, this implies that $F \mapsto\left\langle F, R_{\psi}(x, \cdot)\right\rangle$ is continuous on $L_{p, v}(X)$. Additionally, we require that the weight function $w$ is associated to $v$ in the sense that $\left\{\left\langle F, R_{\psi}(x, \cdot)\right\rangle: F \in L_{p, v}\right\} \subset L_{\infty, 1 / w}$. In [5] natural weight functions $w$ associated to $v$ are proposed. In particular, we can chose $w=1$ if $v \geq 1$. For $1 \leq p \leq \infty$, we define the weighted coorbit spaces

$$
\mathcal{H}_{p, v}:=\left\{f \in \mathcal{H}_{1, w}^{\prime}: V_{\psi} f \in L_{p, v}(X)\right\}
$$

with norms $\|f\|_{\mathcal{H}_{p, v}}:=\left\|V_{\psi} f\right\|_{L_{p, v}}$. The spaces $\left(\mathcal{H}_{p, v},\|\cdot\|_{\mathcal{H}_{p, v}}\right)$ are Banach spaces. Under mild additional conditions it can be shown that $\mathcal{H}_{p, v}$ does not depend on the choice of $\psi$.

Correspondence principle. The basic ingredient in the coorbit theory is a correspondence principle between the spaces $\mathcal{H}_{p, v}$ and certain subspaces of functions on $X$, which are defined by means of the reproducing kernel $R_{\psi}$. For $1 \leq p \leq \infty$ and $R_{\psi}$ with property (3.3), let

$$
\mathcal{M}_{p, v}:=\left\{F \in L_{p, v}(X):\left\langle F, R_{\psi}(x, \cdot)\right\rangle=F(x) \text { a.e. }\right\}
$$


Theorem 2 (Correspondence between $\mathcal{H}_{p, v}$ and $\mathcal{M}_{p, v}$ ). Let $\psi \in \mathcal{H}$ be given such that the corresponding kernel $R_{\psi}$ satisfies (3.3). Then, $V_{\psi} f \in \mathcal{M}_{p, v}$, i.e.,

$$
\left\langle V_{\psi} f, R_{\psi}(x, \cdot)\right\rangle=V_{\psi} f(x), \quad f \in \mathcal{H}_{p, v} .
$$

Conversely, for every $F \in \mathcal{M}_{p, v}, 1 \leq p \leq \infty$, there exists a uniquely determined element $f \in \mathcal{H}_{p, v}$ such that $F=V_{\psi} f$.

By Theorem 2 and (3.2) we see that $V_{\psi} \tilde{V}_{\psi}$ is the identity on $\mathcal{M}_{p, v}$. Since we have for $f \in \mathcal{H}_{p, v}$ that $V_{\psi} f \in \mathcal{M}_{p, v}$ it follows $V_{\psi} \tilde{V}_{\psi} V_{\psi} f=V_{\psi} f$. Now $V_{\psi}$ is injective on $\mathcal{H}_{1, w}^{\prime}$ so that $\tilde{V}_{\psi} V_{\psi}$ is the identity on $\mathcal{H}_{p, v}$.

\section{Atomic Decompositions and Banach Frames}

In this section we show that judicious discretizations of the continuous wavelet transform give rise to atomic decompositions and Banach frames of the form $\left\{\mathcal{U}\left(\sigma\left(x_{i}\right)\right) \psi: x_{i} \in X\right\}$ for weighted coorbit spaces.

Discretizations. A major tool is that of a bounded uniform partition of unity which we adapt to homogeneous spaces. A sequence $\left(x_{i}\right)_{i \in I} \subset X$ is called $U$-dense if $\bigcup_{i \in I} \sigma\left(x_{i}\right) U \supset$ $\sigma(X)$ for some relatively compact neighborhood $U$ of $e \in G$ with nonvoid interior and it is called relatively separated, if $\sup _{j \in I} \#\left\{i \in I: \sigma\left(x_{i}\right) L \cap \sigma\left(x_{j}\right) L \neq \emptyset\right\} \leq C_{L}$ for all compact subsets $L \subset G$. It can be proved that there exist relatively separated and $U$-dense sequences $\left(x_{i}\right)_{i \in I} \subset X$ for all ( $\sigma$-compact) locally compact groups $G$, all closed subgroups $H$ and all relatively compact neighborhoods $U \subset G$ of $e \in G$ with nonvoid interior.

It is standard to construct a bounded partition of unity corresponding to some $U$ dense and relatively separated sequence $\left(x_{i}\right)_{i \in I}$, i.e., a sequence of (continuous) functions $\phi_{i}, i \in I$, on $G$ such that

(a) $0 \leq \phi_{i}(g) \leq 1$ for all $g \in G$,

(b) $\operatorname{supp} \phi_{i} \subset \sigma\left(x_{i}\right) U$,

(c) $\sum_{i \in I} \phi_{i}(\sigma(x))=1$ for all $x \in X$.

We introduce the subsets $X_{i}:=\left\{x \in X: \sigma(x) \in \sigma\left(x_{i}\right) U\right\}$. Clearly, these sets form a covering of $X$ with uniformly finite overlap. In order to carry through the discretization machinery we require for the weight function $v$ that

$$
\frac{v(x)}{v(y)} \leq D \quad \text { for all } x, y \in X_{i}, i \in I
$$

for some constant $D<\infty$ independent of $i \in I$. In the terminology of Feichtinger and Gröbner [12] this means that $v$ is moderate with respect to the covering $\left\{X_{i}\right\}_{i \in I}$.

For simpler notation, we introduce the numbers $a_{i}:=\mu\left(X_{i}\right)$. Let $\ell_{p, v a^{1 / p}}$ denote the space of sequences over $I$ for which

$$
\left\|\left(\eta_{i}\right)_{i \in I}\right\|_{\ell_{p, v a}^{1 / p}}:=\left\|\left(\eta_{i} v\left(x_{i}\right) a_{i}^{1 / p}\right)_{i \in I}\right\|_{\ell_{p}(I)}<\infty .
$$

Clearly, if $\left(a_{i}\right)_{i \in I}$ is bounded from above and below then $\ell_{p, v a^{1 / p}}=\ell_{p, v a^{1 / p-1}}=\ell_{p, v}$ with equivalent norms. In particular, this is the case if $X_{i}=\sigma\left(x_{i}\right) \Pi(U)$. 
Atomic decompositions. For some relatively compact set $U$, we introduce the kernel $\operatorname{osc}_{U}$ dependent on $\psi$

$$
\operatorname{osc}_{U}(x, y):=\sup _{u \in U}\left|\mathcal{U}(\sigma(x))\left(\operatorname{Id}-\mathcal{U}\left(u^{-1}\right)\right) \psi \cdot \mathcal{U}(\sigma(y)) \psi\right\rangle \mid .
$$

The first theorem is a decomposition theorem which says that discretizing the representation by means of a $U$-dense set indeed produces an atomic decomposition of $\mathcal{H}_{p, v}$.

Theorem 3. Let $G$ be a locally compact group with closed subgroup $H$ and let $v$ be a weight function on $X=G / H$. Further, let $\mathcal{U}$ be a square integrable representation of $G$ $\bmod (H, \sigma)$ with strictly admissible function $\psi$. Assume that the kernel $R_{\psi}$ fulfills (3.3) and (3.1) with a weight $w(x) \geq 1$ associated to $v$. Let a relatively compact neighborhood $U$ of the identity in $G$ be chosen such that

$$
\int_{X} \operatorname{osc}_{U}(y, x) \frac{v(x)}{v(y)} d \mu(x) \leq \gamma \quad \text { and } \quad \int_{X} \operatorname{osc}_{U}(y, x) \frac{v(x)}{v(y)} d \mu(y) \leq \gamma,
$$

where $\gamma<1$. Let $\left(x_{i}\right)_{i \in I}$ be a $U$-dense, relatively separated family and assume that $v$ satisfies (4.1). Then $\mathcal{H}_{p, v}, 1 \leq p \leq \infty$, has the following atomic decomposition: If $f \in \mathcal{H}_{p, v}, 1 \leq p \leq \infty$, then $f$ can be represented as

$$
f=\sum_{i \in I} c_{i} \mathcal{U}\left(\sigma\left(x_{i}\right)\right) \psi
$$

where the sequence of coefficients $\left(c_{i}\right)_{i \in I}=\left(c_{i}(f)\right)_{i \in I} \in \ell_{p, v a^{1 / p-1}}$ depends linearly on $f$ and satisfies

$$
\left\|\left(c_{i}\right)_{i \in I}\right\|_{\ell_{p, v a} 1 / p-1} \leq A\|f\|_{\mathcal{H}_{p, v}} .
$$

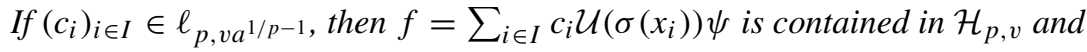

$$
\|f\|_{\mathcal{H}_{p, v}} \leq B\left\|\left(c_{i}\right)_{i \in I}\right\|_{\ell_{p, v a} 1 / p-1} .
$$

Banach frames. Given such an atomic decomposition, the problem arises under which conditions a function $f$ is completely determined by its moments, given by $\langle f$, $\left.\mathcal{U}\left(\sigma\left(x_{i}\right)\right) \psi\right\rangle_{\mathcal{H}_{1, w}^{\prime} \times \mathcal{H}_{1, w}}$, and how $f$ can be reconstructed from these moments. This question is answered by the following theorem which shows that $\left\{\psi_{i}:=\mathcal{U}\left(\sigma\left(x_{i}\right)\right) \psi: i \in I\right\}$ indeed gives rise to a Banach frame.

Theorem 4. Impose the same assumptions as in Theorem 3 with

$$
\int_{X} \operatorname{osc}_{U}(x, y) \frac{v(x)}{v(y)} d \mu(x) \leq \frac{\tilde{\gamma}}{C_{\psi}} \quad \text { and } \quad \int_{X} \operatorname{osc}_{U}(x, y) \frac{v(x)}{v(y)} d \mu(y) \leq \frac{\tilde{\gamma}}{C_{\psi}},
$$

where $\tilde{\gamma}<1$, instead of (4.2).

Then the set

$$
\left\{\psi_{i}:=\mathcal{U}\left(\sigma\left(x_{i}\right)\right) \psi: i \in I\right\}
$$

is a Banach frame for $\mathcal{H}_{p, v}$. This means that

(i) $f \in \mathcal{H}_{p, v}$ if and only if $\left(\left\langle f, \psi_{i}\right\rangle_{\mathcal{H}_{1, w}^{\prime} \times \mathcal{H}_{1, w}}\right)_{i \in I} \in \ell_{p, v a^{1 / p}}$;

(ii) there exist two constants $0<A^{\prime} \leq B^{\prime}<\infty$ such that

$$
A^{\prime}\|f\|_{\mathcal{H}_{p, v}} \leq\left\|\left(\left\langle f, \psi_{i}\right\rangle_{\mathcal{H}_{1, w}^{\prime} \times \mathcal{H}_{1, w}}\right)_{i \in I}\right\|_{\ell_{p, v a} 1 / p} \leq B^{\prime}\|f\|_{\mathcal{H}_{p, v}} ;
$$

(iii) there exists a bounded, linear reconstruction operator $\mathcal{S}$ from $\ell_{p, v a^{1 / p}}$ to $\mathcal{H}_{p, v}$ such that $\mathcal{S}\left(\left(\left\langle f, \psi_{i}\right\rangle_{\mathcal{H}_{1, w}^{\prime}} \times \mathcal{H}_{1, w}\right)_{i \in I}\right)=f$. 
Approximation operators. Let us briefly explain the basic concept of the proofs of Theorems 3 and 4. The main ingredient is that the operator which maps $F \in \mathcal{M}_{p, v}$ onto the function $\left\langle F, R_{\psi}(x, \cdot)\right\rangle$ is the identity on $\mathcal{M}_{p, v}$. The idea now is to approximate this operator which is given by an integral by a sum. As in $[4,5]$ we use the following two approximation operators

$$
\begin{aligned}
T_{\phi} F(x) & :=\sum_{i \in I}\left\langle F, \phi_{i} \circ \sigma\right\rangle R_{\psi}\left(x_{i}, x\right) \\
& =\sum_{i \in I} \int_{X} F(y) \phi_{i}(\sigma(y)) d \mu(y) R_{\psi}\left(x_{i}, x\right), \\
S_{\phi} F(x) & :=\sum_{i \in I} F\left(x_{i}\right)\left\langle\phi_{i} \circ \sigma, R_{\psi}(x, \cdot)\right\rangle \\
& =\sum_{i \in I} \int_{X} F\left(x_{i}\right) \phi_{i}(\sigma(y)) R_{\psi}(y, x) d \mu(y) .
\end{aligned}
$$

We have to prove that these operators are invertible under certain conditions, see Theorem 5 below. Then the correspondence principle (Theorem 2) combined with the operators $T_{\phi}$ and $S_{\phi}$ yields an atomic decomposition and a Banach frame, respectively.

We shall only briefly explain how the operator $T_{\phi}$ can be used to obtain the atomic decomposition (4.3). For a detailed discussion on the operator $S_{\phi}$ we again refer to [4, 5]. Assume $f \in \mathcal{H}_{p, v}$ so that $V_{\psi} f \in L_{p, v}$. If the operator $T_{\phi}$ is boundedly invertible, we obtain

$$
V_{\psi} f(x)=T_{\phi} T_{\phi}^{-1} V_{\psi} f(x)=\sum_{i \in I}\left\langle T_{\phi}^{-1} V_{\psi} f, \phi_{i} \circ \sigma\right\rangle R_{\psi}\left(x_{i}, x\right) .
$$

Since $R_{\psi}\left(x_{i}, x\right)=V_{\psi}\left(U\left(\sigma\left(x_{i}\right) \psi\right)\right)(x)$ and $\tilde{V}_{\psi} V_{\psi}$ is the identity on $\mathcal{H}_{p, v}$, Equation (4.7) yields

$$
f=\tilde{V}_{\psi}\left(\sum_{i \in I} c_{i}(f) V_{\psi} U\left(\sigma\left(x_{i}\right)\right) \psi\right)
$$

with $c_{i}(f):=\left\langle T_{\phi}^{-1} V_{\psi} f, \phi_{i} \circ \sigma\right\rangle$. As $\tilde{V}_{\psi}$ is continuous on $\mathcal{H}_{p, v}$, we obtain

$$
f=\sum_{i \in I} c_{i}(f) U\left(\sigma\left(x_{i}\right)\right) \psi .
$$

For the proof of the frame bounds (4.4), (4.5) we refer again to $[4,5]$.

\section{Theorem 5.}

(i) Suppose that there exists $\gamma<1$ such that (4.2) holds. Then $\left\|\mathrm{Id}-T_{\phi}\right\|_{\mathcal{M}_{p, v} \rightarrow \mathcal{M}_{p, v}} \leq$ $\gamma<1$. In particular, $T_{\phi}$ is bounded with bounded inverse.

(ii) Suppose that $R$ fulfills (3.3) and that there exists $\tilde{\gamma}<1$ such that (4.6) holds where $C_{\psi}$

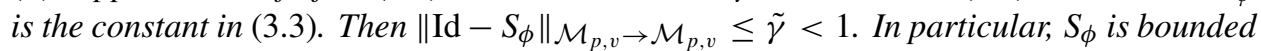
with bounded inverse.

Proof. We only sketch the proof of part (i), the second part can be proved analogously. Using the reproducing formula on $\mathcal{M}_{p, v}$ and the fact that $\left(\phi_{i} \circ \sigma\right)_{i \in I}$ is a partition of unity on $X$ we obtain for $F \in \mathcal{M}_{p, v}$

$$
F(x)=\int_{X} F(y) \overline{R_{\psi}(x, y)} d \mu(y)=\sum_{i \in I} \int_{X} F(y) \phi_{i}(\sigma(y)) R_{\psi}(y, x) d \mu(y) .
$$


It follows immediately that

$$
F(x)-T_{\phi} F(x)=\sum_{i \in I} \int_{X} F(y) \phi_{i}(\sigma(y))\left[R_{\psi}(y, x)-R_{\psi}\left(x_{i}, x\right)\right] d \mu(y) .
$$

By definition of $R$ we obtain

$$
\begin{aligned}
& \left|F(x)-T_{\phi} F(x)\right| \leq \sum_{i \in I} \int_{X}|F(y)| \phi_{i}(\sigma(y))\left|R_{\psi}(y, x)-R_{\psi}\left(x_{i}, x\right)\right| d \mu(y) \\
& \quad=\sum_{i \in I} \int_{X}|F(y)| \phi_{i}(\sigma(y))\left|\left\langle\left(\mathcal{U}(\sigma(y))-\mathcal{U}\left(\sigma\left(x_{i}\right)\right)\right) \psi, \mathcal{U}(\sigma(x)) \psi\right\rangle\right| d \mu(y) .
\end{aligned}
$$

Since supp $\phi_{i} \subset \sigma\left(x_{i}\right) U$ we are only interested in those $y \in X$ such that $\sigma(y) \in \sigma\left(x_{i}\right) U$ which implies $\sigma(y)=\sigma\left(x_{i}\right) u$ for some $u \in U$ or equivalently $\sigma\left(x_{i}\right)=\sigma(y) u^{-1}$. Hence, we have

$$
\begin{aligned}
& \left|F(x)-T_{\phi} F(x)\right| \\
& \quad \leq \sum_{i \in I} \int_{X}|F(y)| \phi_{i}(\sigma(y)) \sup _{u \in U}\left|\left\langle\left(\mathcal{U}(\sigma(y))-\mathcal{U}\left(\sigma(y) u^{-1}\right)\right) \psi, \mathcal{U}(\sigma(x)) \psi\right\rangle\right| d \mu(y) \\
& \quad=\sum_{i \in I} \int_{X}|F(y)| \phi_{i}(\sigma(y)) \operatorname{osc}_{U}(y, x) d \mu(y)=\int_{X}|F(y)| \operatorname{osc}_{U}(y, x) d \mu(y) .
\end{aligned}
$$

By (4.2) and the generalized Young inequality, see Theorem A.1, we obtain

$$
\left\|F-T_{\phi} F\right\|_{\mathcal{M}_{p, v}}=\left\|\left(\operatorname{Id}-T_{\phi}\right) F\right\|_{\mathcal{M}_{p, v}} \leq \gamma\|F\|_{\mathcal{M}_{p, v}} .
$$

Hence, $\left\|\operatorname{Id}-T_{\phi}\right\|_{\mathcal{M}_{p, v} \rightarrow \mathcal{M}_{p, v}} \leq \gamma<1$ and thus $T_{\phi}$ is boundedly invertible on $\mathcal{M}_{p, v}$.

\section{Nonlinear Approximation}

The established atomic decomposition can now be used to decompose, to approximate and to analyze certain functions on $\mathcal{H}_{p, v}$. Then it is clearly desirable to determine the quality of certain approximation schemes based on our atomic decomposition, i.e., the approximation order comes into play. In this section, we are interested in the quality of the best $N$-term approximation. We restrict ourselves to the case where the sequence $\left(a_{i}\right)_{i \in I}$ is bounded from below and from above. This is the case if the measure under consideration is invariant and not only quasi-invariant. Fortunately, for the application we have in mind this property is indeed satisfied, see Section 6.1.

The setting can be described as follows. Let $\left\{\psi_{i}=U\left(\sigma\left(x_{i}\right)\right) \psi: i \in I\right\}$ denote the set of atomic functions constructed in the previous section, i.e., we have for any $f \in$ $\mathcal{H}_{p, v}$ that

$$
f=\sum_{i \in I} c_{i} \psi_{i}
$$

and

$$
\left\|\left(c_{i}\right)_{i \in I}\right\|_{\ell_{p, v}} \sim\|f\|_{\mathcal{H}_{p, v}} .
$$


We want to approximate our functions $f \in \mathcal{H}_{p, v}$ by elements from the nonlinear manifolds $\Sigma_{n}, n \in \mathbb{N}$, which consist of all functions $S \in \mathcal{H}_{p, v}$ whose expansions with respect to our discrete coherent states have at most $n$ nonzero coefficients, i.e.,

$$
\Sigma_{n}:=\left\{S \in \mathcal{H}_{p, v}: S=\sum_{i \in J} b_{i} \psi_{i}, J \subseteq I, \operatorname{card} J \leq n\right\}
$$

Then we are interested in the asymptotic behavior of the error

$$
E_{n}(f)_{\mathcal{H} p, v}:=\inf _{S \in \Sigma_{n}}\|f-S\|_{\mathcal{H}_{p, v}} .
$$

Usually, the order of approximation which can be achieved depends on the regularity of the approximated function as measured in some associated smoothness space. For instance, for nonlinear wavelet approximation, the order of convergence is determined by the regularity as measured in a specific scale of Besov spaces. For nonlinear approximation based on Gabor frames, it has been shown in [16] that the 'right' smoothness spaces are given by a specific scale of modulation spaces. It turns out that the result from [16], i.e., an upper estimate, carries over to our case without any difficulty. The basic ingredient in the proof of the theorem is the following lemma which has been shown in [16], see also [7].

Lemma 1. Let $a=\left(a_{i}\right)_{i=1}^{\infty}$ be a decreasing sequence of positive numbers. For $p, q>0$ set $\alpha:=1 / p-1 / q$ and $E_{n, q}(a):=\left(\sum_{i=n}^{\infty} a_{i}^{q}\right)^{1 / q}$. Then for $0<p<q \leq \infty$ we have

$$
2^{-1 / p}\|a\|_{\ell_{p}} \leq\left(\sum_{n=1}^{\infty}\left(n^{\alpha} E_{n, q}(a)\right)^{p} \frac{1}{n}\right)^{1 / p} \leq C\|a\|_{\ell_{p}}
$$

with a constant $C>0$ depending only on $p$.

Now one can prove the following theorem, see also [16].

Theorem 6. Let $\left\{\psi_{i}: i \in I\right\}$ be a set of atomic functions for $\mathcal{H}_{p, v}, 1 \leq p \leq \infty$, as constructed by Theorem 3. If $1 \leq p<q, \alpha:=1 / p-1 / q$ and $f \in \mathcal{H}_{p, v}$, then

$$
\left(\sum_{n=1}^{\infty} \frac{1}{n}\left(n^{\alpha} E_{n}(f)_{\mathcal{H}_{q, v}}\right)^{p}\right)^{1 / p} \leq C\|f\|_{\mathcal{H}_{p, v}}
$$

for a constant $C<\infty$. 


\section{Proof.}

Let $\pi$ permutate the sequence $\left(c_{i}\right)_{i \in I}$ in (5.1) in a decreasing order, i.e., $\left|c_{\pi(1)} v\left(x_{\pi(1)}\right)\right| \geq\left|c_{\pi(2)} v\left(x_{\pi(2)}\right)\right| \geq \ldots$ Then we obtain

$$
E_{n}(f)_{\mathcal{H}_{q, v}} \leq\left\|\sum_{i=n+1}^{\infty} c_{\pi(i)} \psi_{\pi(i)}\right\|_{\mathcal{H}_{q, v}}
$$

and by (5.2) further that

$$
\begin{aligned}
E_{n}(f)_{\mathcal{H}_{q, v}} & \leq C\left(\sum_{i=n+1}^{\infty}\left|c_{\pi(i)}\right|^{q} v\left(x_{\pi(i)}\right)^{q}\right)^{1 / q} \\
& =C E_{n+1, q}\left(\left|c_{\pi(i)}\right| v\left(x_{\pi(i)}\right)\right) \leq C E_{n, q}\left(\left|c_{\pi(i)}\right| v\left(x_{\pi(i)}\right)\right) .
\end{aligned}
$$

Now we finish by applying Lemma 1 and (5.2)

$$
\begin{aligned}
\left(\sum_{n=1}^{\infty} \frac{1}{n}\left(n^{\alpha} E_{n}(f)_{\mathcal{H}_{q, v}}\right)^{p}\right)^{1 / p} & \leq\left(\sum_{n=1}^{\infty} \frac{1}{n}\left(n^{\alpha} C E_{n, q}\left(\left|c_{\pi(i)}\right| v\left(x_{\pi(i)}\right)\right)\right)^{p}\right)^{1 / p} \\
& \left.\leq C^{\prime} \|\left(\left|c_{\pi(i)}\right| v\left(x_{\pi(i)}\right)\right)\right)\left\|_{\ell_{p}}=C^{\prime}\right\| c \|_{\ell_{p, v}} \\
& \leq C^{\prime \prime}\|f\|_{\mathcal{H}_{p, v}} .
\end{aligned}
$$

\section{Application to the Sphere}

In this section, we want to fill our technical consideration with live by deriving a generalized windowed Fourier transform on the spheres $S^{n-1}$ and checking that the proposed construction of weighted modulation spaces and Banach frames works well for this setting.

\subsection{Modulation Spaces and Banach Frames}

We start by establishing a suitable group representation for the Hilbert space $\mathcal{H}=L_{2}\left(S^{n-1}\right)$. Having the usual windowed Fourier transform generated by translations and modulations in mind, Torresani suggested in [18] to choose the Euclidean group $G:=E(n)=S O(n) \ltimes \mathbb{R}^{n}$, with group operation

$$
(R, p) \circ(\tilde{R}, \tilde{p})=(\tilde{R} R, \tilde{R} p+\tilde{p}), \quad(R, p)^{-1}=\left(R^{-1},-R^{-1} p\right) .
$$

As a natural analogue to the Schrödinger representation of the Weyl-Heisenberg group on $L_{2}\left(\mathbb{R}^{n}\right)$, we can define the continuous unitary representation

$$
\mathcal{U}(R, p) f(s):=e^{-i<R s, p>} f(R s), \quad s \in S^{n-1}
$$

of $G$ on $\mathcal{H}$. Since this representation is not square integrable, we are looking for suitable representations modulo a subgroup $H$ of $G$.

In order to keep the notation simple, we restrict ourselves to the case $\mathcal{H}=L_{2}\left(S^{1}\right) \cong$ $L_{2}([-\pi, \pi])$. In this setting, $R \in S O(2)$ and $s \in S^{1}$ are given explicitly by

$$
R=\left(\begin{array}{rr}
\cos \theta & \sin \theta \\
-\sin \theta & \cos \theta
\end{array}\right), \quad s=\left(\begin{array}{c}
\sin \gamma \\
\cos \gamma
\end{array}\right) .
$$


To overcome the integrability problem, we use the subgroup $H:=\left\{\left(0,0, p_{2}\right): p_{2} \in \mathbb{R}\right\}$ of $G$ together with the flat section $\sigma\left(\theta, p_{1}\right):=\left(\theta, p_{1}, 0\right)$. Then $X:=G / H$ carries the $G$ invariant measure $d \mu(x)=d p_{x} d \theta_{x}$, where $x=\left(\theta_{x}, p_{x}, 0\right)$. In this case, the representation reads as

$$
\mathcal{U}\left(\sigma\left(\theta, p_{1}\right)\right) f(\gamma)=e^{-i \sin (\gamma+\theta) p_{1}} f(\gamma+\theta) .
$$

The following lemma ensures strictly square integrability of $\mathcal{U} \bmod (H, \sigma)$.

Lemma 2. Assume that the function $\psi \in L_{2}([-\pi, \pi])$ is such that $\operatorname{supp} \psi \subset[-\pi / 2, \pi / 2]$ and

$$
2 \pi \int_{-\pi / 2}^{\pi / 2} \frac{|\psi(\gamma)|^{2}}{\cos \gamma} d \gamma=1
$$

Then the map $V_{\psi}$ defined by (2.1) is an isometry.

The proof in [18] uses that

$$
V_{\psi} f(x)=\left\langle\mathcal{U}\left(\sigma(x)^{-1}\right) f, \psi\right\rangle=\int_{-\pi / 2}^{\pi / 2} e^{i p_{x} \sin \gamma} f\left(\gamma-\theta_{x}\right) \bar{\psi}(\gamma) d \gamma .
$$

As a consequence of the theorem, the wavelet transform can be inverted by using the adjoint $V_{\psi}^{*}$. Of course the approach works also if

$$
0<c_{\psi}:=2 \pi \int_{-\pi / 2}^{\pi / 2} \frac{|\psi(\gamma)|^{2}}{\cos \gamma} d \gamma<\infty
$$

Then the inverse of the wavelet transform is given by $V_{\psi}^{*} / \sqrt{c_{\psi}}$.

In the following, we choose the admissible function

$$
\psi(\gamma)=\cos ^{6} \gamma \cdot \chi_{[-\pi / 2, \pi / 2]}(\gamma) .
$$

For $x=\left(\theta_{x}, p_{x}, 0\right), y=\left(\theta_{y}, p_{y}, 0\right) \in X$ and $\theta=\theta_{x}-\theta_{y}$ we see as in [3] that the kernel $R_{\psi}$ can be rewritten as

$$
R_{\psi}(y, x)=\hat{F}_{\theta, p_{y}}\left(-p_{x}\right),
$$

where

$$
F_{\theta, p_{y}}(t):=e^{-i p_{y} \sin (\arcsin t-\theta)} \psi(\arcsin t-\theta) \bar{\psi}(\arcsin t) / \sqrt{1-t^{2}} .
$$

The plots of $\left|R_{\psi}(x, y)\right|=\left|\hat{F}_{\theta, p_{y}}\left(-p_{x}\right)\right|$ for two values of $\theta$ in Figure 1 describe the typical decay behavior of $R_{\psi}$.

In analogy to the classical modulation spaces on the Euclidean plane, we consider specific weight functions of the form $v\left(\theta, p_{1}\right)=\left(1+\left|p_{1}\right|\right)^{s}, s>0$ i.e., the modulation spaces are generalized Bessel-potential spaces. To $v$ we associate $w=1$. In order to construct properly defined weighted modulation spaces we have to establish the fundamental properties (3.3) of our kernel $R_{\psi}$. To this end, we use that

$$
\int_{X}|R(x, y)| \frac{w(x)}{w(y)} d \mu(x)=\int_{-\pi}^{\pi} \int_{\mathbb{R}}\left|\hat{F}_{\theta, p_{y}}\left(p_{x}\right)\right| \frac{\left(1+\left|p_{x}\right|\right)^{s}}{\left(1+\left|p_{y}\right|\right)^{s}} d p_{x} d \theta_{x} .
$$



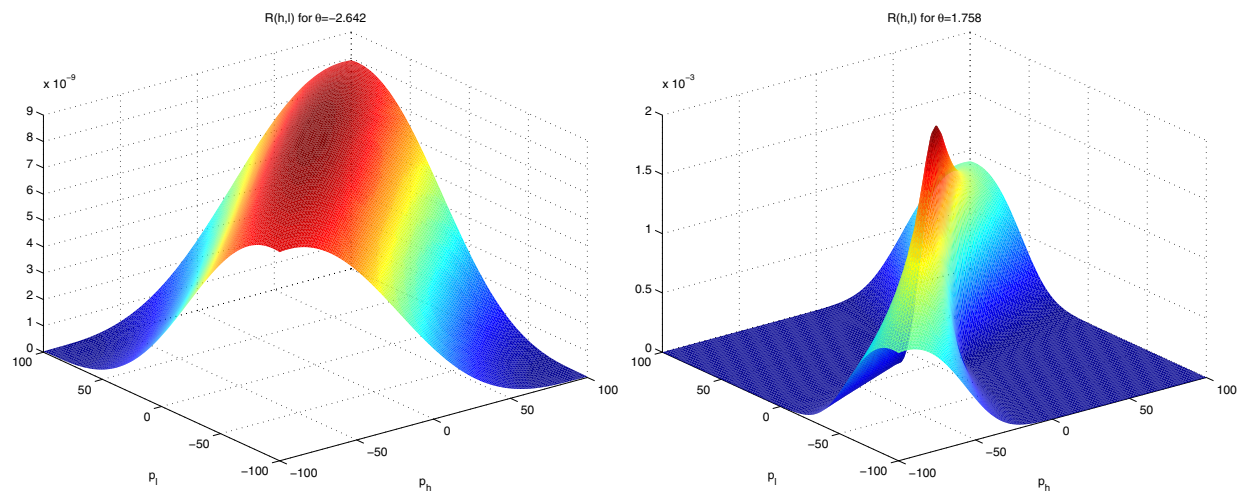

FIGURE $1 \quad$ Left: $\left|\hat{F}_{\theta, p_{y}}\left(-p_{x}\right)\right|$ for $\theta=-2.642$, right: $\left|\hat{F}_{\theta, p_{y}}\left(-p_{x}\right)\right|$ for $\theta=1.758$.

Regarding that the outer integration is over a finite interval it remains to check that

$$
\int_{\mathbb{R}}\left|\hat{F}_{\theta, p_{y}}\left(p_{x}\right)\right| \frac{\left(1+\left|p_{x}\right|\right)^{s}}{\left(1+\left|p_{y}\right|\right)^{s}} d p_{x} \leq C, \quad \text { and } \int_{\mathbb{R}}\left|\hat{F}_{\theta, p_{y}}\left(p_{x}\right)\right| \frac{\left(1+\left|p_{x}\right|\right)^{s}}{\left(1+\left|p_{y}\right|\right)^{s}} d p_{y} \leq C
$$

with some constants $C$ independent of $\theta$ and $p_{y}$ and $p_{x}$, respectively.

These properties are confirmed numerically and the results are presented in the Figures $2-4$ for $s=0.5$. Figure 2 shows the approximated values of $\int_{\mathbb{R}}\left|\hat{F}_{\theta, p_{y}}\left(p_{x}\right)\right|(1+$ $\left.\left|p_{x}\right|\right)^{0.5} /\left(1+\left|p_{y}\right|\right)^{0.5} d p_{x}$ as functions of $p_{y}$ and Figure 3 the approximated values of $\int_{\mathbb{R}}\left|\hat{F}_{\theta, p_{y}}\left(p_{x}\right)\right|\left(1+\left|p_{x}\right|\right)^{0.5} /\left(1+\left|p_{y}\right|\right)^{0.5} d p_{y}$ as functions of $p_{x}$. Finally, in Figure 4 , we have displayed $\max _{p_{y}} \int \mid \hat{F}_{\theta, p_{y}}\left(p_{x}\right)\left(1+\left|p_{x}\right|\right)^{0.5} /\left(1+\left|p_{y}\right|\right)^{0.5} d p_{x}$ and $\max _{p_{x}} \int \mid \hat{F}_{\theta, p_{y}}\left(p_{x}\right)(1+$ $\left.\left|p_{x}\right|\right)^{0.5} /\left(1+\left|p_{y}\right|\right)^{0.5} d p_{y}$ for all $\theta \in[-\pi, \pi]$. These results clearly show that conditions (6.3) are satisfied.
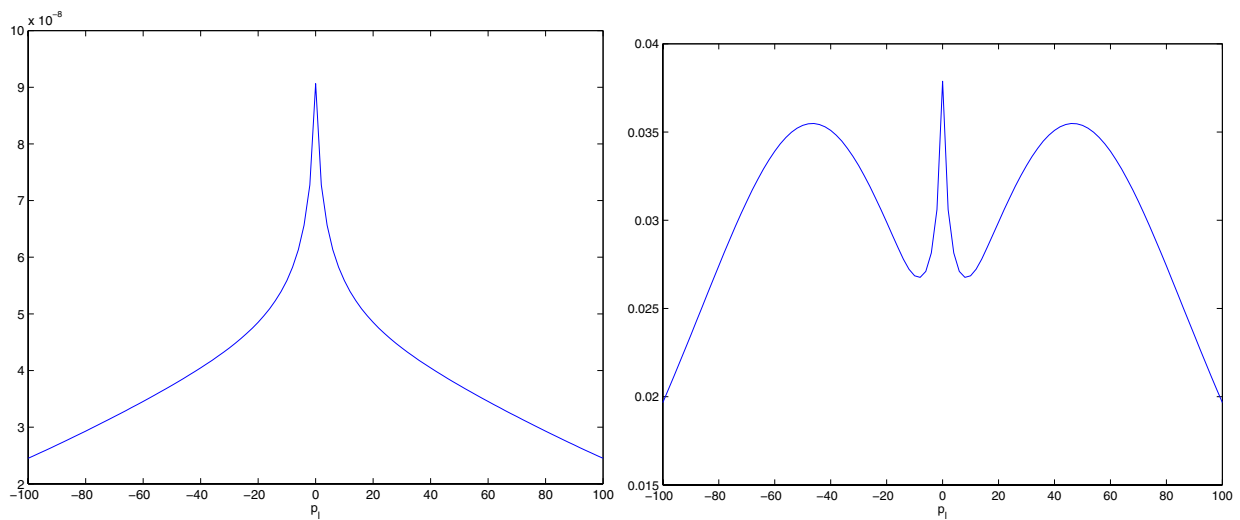

FIGURE $2 \int_{\mathbb{R}}\left|\hat{F}_{\theta, p_{y}}\left(p_{x}\right)\right|\left(1+\left|p_{x}\right|\right)^{0.5} /\left(1+\left|p_{y}\right|\right)^{0.5} d p_{x}$, left: $\theta=2.642$, right: $\theta=1.758$.

For the construction of Banach frames in $\mathcal{M}_{p, v}$ we choose the neighborhood $U:=$ $[-\pi / N, \pi / N] \times[-\pi / M, \pi / M] \times[-\pi / M, \pi / M]$ of the identity and a $U$-dense set $\left(x_{n, m}\right)_{(n, m) \in I}$ with $x_{n, m}=\left(\theta_{n}, p_{m}, q_{m}\right)$. Then the assumptions concerning $\operatorname{osc}_{U}$ in Theorem 3 and Theorem 4 , respectively, can be verified directly by slightly modifying the steps 

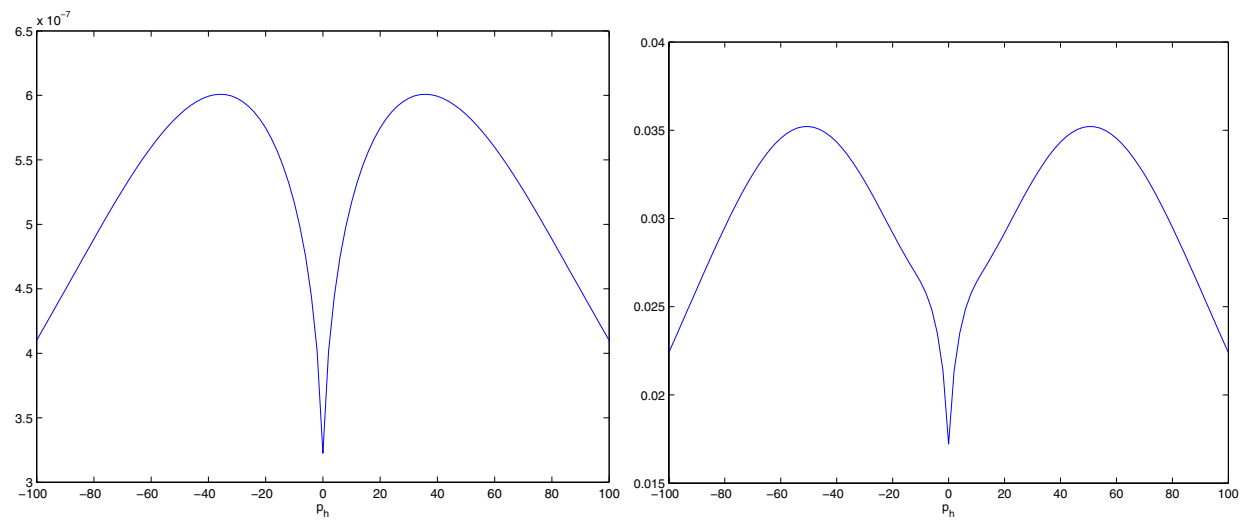

FIGURE $3 \int_{\mathbb{R}}\left|\hat{F}_{\theta, p_{y}}\left(p_{x}\right)\right|\left(1+\left|p_{h}\right|\right)^{0.5} /\left(1+\left|p_{y}\right|\right)^{0.5} d p_{y}$, left: $\theta=2.642$, right: $\theta=1.758$.
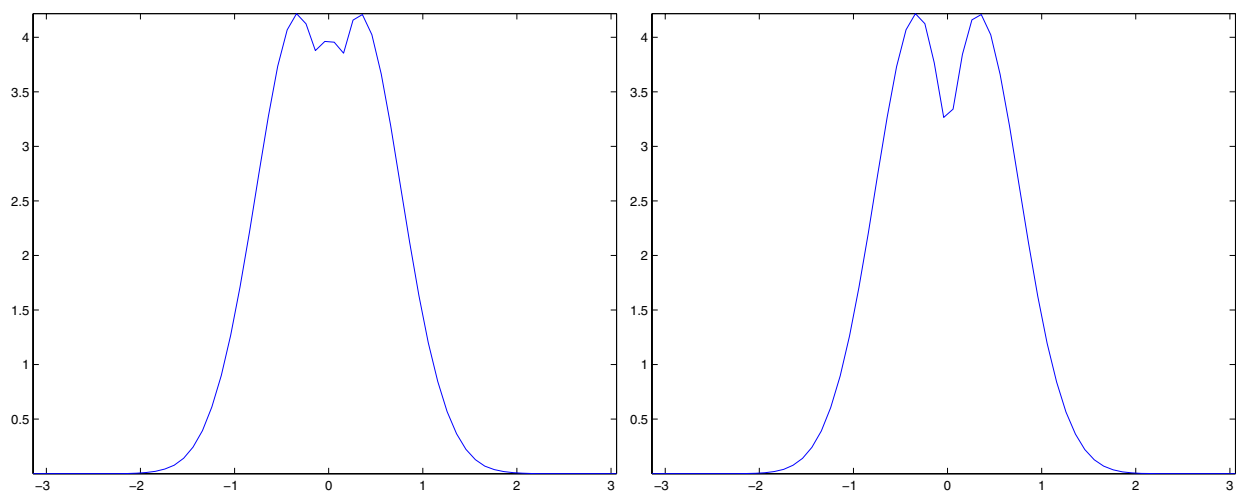

FIGURE 4 Maximum plot for all $\theta \in[-\pi, \pi]$; left: $\max _{p_{y}} \int_{\mathbb{R}}\left|\hat{F}_{\theta, p_{y}}\left(p_{x}\right)\right|\left(1+\left|p_{x}\right|\right)^{0.5} /\left(1+\left|p_{y}\right|\right)^{0.5} d p_{x}$, right: $\max _{p_{x}} \int_{\mathbb{R}}\left|\hat{F}_{\theta, p_{y}}\left(p_{x}\right)\right|\left(1+\left|p_{x}\right|\right)^{0.5} /\left(1+\left|p_{y}\right|\right)^{0.5} d p_{y}$.

in [3] with respect to the additional weight function.

\subsection{Time-Frequency Localized Analyzing Atmos}

A general theorem which is well-known in quantum mechanics and harmonic analysis [13] relates an uncertainty principle to any two self-adjoint operators and provides a mechanism for deriving a minimizing function for the uncertainty relation. Before repeating this wellknown result on uncertainties, let us fix some notation. Let $A_{1}, A_{2}$ be two self-adjoint operators. Their commutator is defined by

$$
\left[A_{1}, A_{2}\right]:=A_{1} A_{2}-A_{2} A_{1},
$$

the expectation of some operator $A_{1}$ with respect to some state $\varphi \in \operatorname{dom}\left(A_{1}\right)$, with $\|\varphi\|=$ 1 , by

$$
\mu\left(A_{1}\right):=\mu_{A_{1}}:=\left\langle A_{1} \varphi, \varphi\right\rangle
$$

and, finally, the variance of $A_{1}$ with respect to some state $\varphi \in \operatorname{dom}\left(A_{1}\right)$ by

$$
\left(\Delta A_{1}\right)^{2}:=\mu\left(\left(A_{1}-\mu\left(A_{1}\right)\right)^{2}\right) .
$$


Theorem 7. Given two self-adjoint operators $A_{1}$ and $A_{2}$, then for all $\varphi \in \operatorname{dom}\left(A_{1}\right) \cap$ $\operatorname{dom}\left(A_{2}\right)$ they obey the uncertainty relation:

$$
\Delta A_{1} \Delta A_{2} \geq \frac{1}{2}\left|\left\langle\left[A_{1}, A_{2}\right]\right\rangle\right|,
$$

where $\left|\left\langle\left[A_{1}, A_{2}\right]\right\rangle\right|:=\left|\left\langle\left[A_{1}, A_{2}\right] \varphi, \varphi\right\rangle\right|$. A state $\psi$ is said to have minimal uncertainty if the above inequality turns into an equality. This happens iff there exists an $\lambda \in i \mathbb{R}$ such that

$$
\left(A_{1}-\mu_{A_{1}}\right) \varphi=\lambda\left(A_{2}-\mu_{A_{2}}\right) \varphi .
$$

Suppose that we are given a unitary representation of a Lie group. The linearized operation of the group at the identity element can be described by the infinitesimal generators of the related Lie algebra. If the group representation is unitary, then the infinitesimal generators can transformed to be self-adjoint operators. Thus, the general uncertainty theorem stated above provides a tool for obtaining uncertainty principles using these infinitesimal generators. In the case of the Weyl-Heisenberg group, the canonical functions that minimize the corresponding uncertainty relation are Gaussian functions. The canonical functions that minimize the uncertainty relations for the affine group in one dimension and for the similitude group in two dimensions, were the subject of the previous studies $[1,2]$.

In this section, we want to compute the canonical minimizing states for the local Fourier transform on the sphere as introduced in Section 6.1. Since we are working with quotient manifolds, we are usually loosing the group structure, but nevertheless, in the case of the flat section, there is a canonical substitute for the identity element, namely $\left(\theta, p_{1}\right)=(0,0)$. Since the representation is smooth, we may compute the derivatives at this point to obtain generalized infinitesimal generators.

Theorem 8. The infinitesimal operators $A_{\theta}, A_{p_{1}}$ associated with the local Fourier transform on the sphere are given by

$$
\left(A_{\theta} \varphi\right)(\gamma)=i \varphi^{\prime}(\gamma), \quad \text { and } \quad\left(A_{p_{1}} \varphi\right)(\gamma)=\sin \gamma \varphi(\gamma) .
$$

The state $\varphi$ which is the minimizer of the associated uncertainty is of the form

$$
\varphi(\gamma)=c e^{t \cos \gamma-i \mu_{\theta} \gamma}
$$

where $t \in \mathbb{R}, \mu_{\theta}:=\mu_{A_{\theta}}$, and $c$ has to be chosen that such $\|\varphi\|_{L_{2}\left(S^{1}\right)}=1$.

Proof. Taking the derivatives with respect to $\theta$ and $p_{1}$ in (6.1) and evaluating them at $\theta=0, p_{1}=0$ leads to

$$
\begin{aligned}
\left.\frac{\partial}{\partial \theta} \mathcal{U}\left(\sigma\left(\theta, p_{1}\right)\right) \varphi\right|_{\left(\theta, p_{1}\right)=(0,0)}(\gamma) & =\varphi^{\prime}(\gamma) \\
\left.\frac{\partial}{\partial p_{1}} \mathcal{U}\left(\sigma\left(\theta, p_{1}\right)\right) \varphi\right|_{\left(\theta, p_{1}\right)=(0,0)}(\gamma) & =-i \sin \gamma \varphi(\gamma) .
\end{aligned}
$$

These operators are not self-adjoint, but multiplication with the imaginary unit $i$ yields self-adjoint operators $A_{\theta}=i \frac{\partial}{\partial \theta} \mathcal{U}$ and $A_{p_{1}}=i \frac{\partial}{\partial p_{1}} \mathcal{U}$. This proves (6.6).

The commutator between these two operators is nonzero. By means of Theorem 7, we may calculate those states that minimize the corresponding uncertainty principle. Indeed, (6.5) provides us with the differential equation

$$
\varphi^{\prime}(\gamma)=-i \varphi(\gamma)\left(\lambda \sin \gamma-\lambda \mu_{p_{1}}+\mu_{\theta}\right) .
$$


Now (6.8) can be solved by separation of variables which leads with $\lambda=i t, t \in \mathbb{R}$ to

$$
\varphi(\gamma)=c e^{-t \cos \gamma-\left(i \mu_{\theta}+t \mu_{p_{1}}\right) \gamma} .
$$

However, since we need periodic functions, we necessarily have to choose $\mu_{p_{1}}=0$. This proves (6.7).

Unfortunately, the conditions of Lemma 2 and Theorem 8 cannot be satisfied at the same time, i.e., the minimizing states are not admissible. Nevertheless, we may define canonical admissible vectors $\psi$ supported on $[-\pi / 2, \pi / 2]$ which fit $\varphi$ in the least squares sense and fulfill the admissibility condition (6.2). To this end, let us consider the strictly convex functional

$$
\Phi(\psi):=\alpha \int_{-\pi / 2}^{\pi / 2} \frac{|\psi(\gamma)|^{2}}{\cos \gamma} d \gamma+\int_{-\pi / 2}^{\pi / 2}|\psi(\gamma)-\varphi(\gamma)|^{2} d \gamma,
$$

and compute the minimizers.

Theorem 9. The minimizer of the functional $\Phi(\psi)$ is given by

$$
\psi(\gamma)=\varphi(\gamma) \frac{\cos \gamma}{\cos \gamma+\alpha} \chi_{[-\pi / 2, \pi / 2]}(\gamma) .
$$

Proof. Setting the first variation of (6.9) to zero we obtain the necessary and sufficient minimum condition

$$
\begin{aligned}
& \alpha \int_{-\pi / 2}^{\pi / 2} \frac{\psi(\gamma) \bar{h}(\gamma) \bar{\psi}(\gamma) h(\gamma)}{\cos \gamma} d \gamma \\
& \quad+\int_{-\pi / 2}^{\pi / 2}\{(\psi(\gamma)-\varphi(\gamma)) \bar{h}(\gamma)+h(\gamma)(\bar{\psi}(\gamma)-\bar{\varphi}(\gamma))\} d \gamma=0
\end{aligned}
$$

for all $h \in L_{2}\left(S^{1}\right)$ which is satisfied if

$$
\alpha \frac{\psi(\gamma)}{\cos \gamma}=\varphi(\gamma)-\psi(\gamma), \quad \text { i.e., } \quad \psi(\gamma)=\varphi(\gamma) \frac{\cos \gamma}{\cos \gamma+\alpha} .
$$

\section{Appendix}

\section{A. Appendix}

The generalized Young inequality for $L_{p, v}$ is a major tool in our considerations.

Theorem A.1. Let $K$ be some kernel on $X \times X$. We associate to $K$ the integral operator

$$
K(F)(x):=\int_{X} K(x, y) F(y) d \mu(y)
$$

If $K$ satisfies

$$
\text { ess } \sup _{x \in X} \int_{X}|K(x, y)| \frac{v(x)}{v(y)} d \mu(y) \leq C_{K}<\infty,
$$


then $K$ is a continuous operator on $L_{\infty, v}(X)$. If $K$ satisfies

$$
\text { ess } \sup _{y \in X} \int_{X}|K(x, y)| \frac{v(x)}{v(y)} d \mu(x) \leq C_{K}<\infty \text {, }
$$

then $K$ is a continuous operator on $L_{1, v}(X)$. If $K$ satisfies both (A.1) and (A.2) then $K$ is a continuous operator on $L_{p, v}(X), 1 \leq p \leq \infty$, and satisfies

$$
\|K(F)\|_{L_{p, v}(X)} \leq C_{K}\|F\|_{L_{p, v}(X)} .
$$

\section{References}

[1] Ali, S. T., Antoine, J.-P., and Gazeau, J.-P. (2000). Coherent States, Wavelets and Their Generalizations, Springer-Verlag, New York.

[2] Dahlke, S. and Maass, P. (1995). The affine uncertainty principle in one and two dimensions, Comput. Math. Appl. 30(3-6), 293-305.

[3] Dahlke, S., Steidl, G., and Teschke, G. (2004). Coorbit spaces and Banach frames on homogeneous spaces with applications to analyzing functions on spheres, Adv. Comput. Math. 21, 147-180.

[4] Dahlke, S., Steidl, G., and Teschke, G. (2004). Weighted coorbit spaces and Banach frames on homogeneous spaces, J. Fourier Anal. Appl. 10(5), 507-539.

[5] Dahlke, S., Fornasier, M., Rauhut, H., Steidl, G., and Teschke, G. (2005). Generalized Coorbit Theory, Banach Frames, and the Relation to $\alpha$-Modulation Spaces, Bericht Nr. 2005-6, Philis-University of Marburg.

[6] DeVore, R. (1998). Nonlinear approximation, Acta Num. 7, 51-150.

[7] DeVore, R. and Temlyakov, V. N. (1996). Some remarks on greedy algorithms, Adv. Comput. Math. 5, $173-$ 187.

[8] Feichtinger, H. G. and Gröchenig, K. (1988). A unified approach to atomic decompositions via integrable group representations, Proc. Conf. "Function Spaces and Applications," Lund 1986, Lecture Notes in Math. 1302, 52-73.

[9] Feichtinger, H. G. and Gröchenig, K. (1989). Banach spaces related to integrable group representations and their atomic decomposition I, J. Funct. Anal. 86, 307-340.

[10] Feichtinger, H. G. and Gröchenig, K. (1989). Banach spaces related to integrable group representations and their atomic decomposition II, Monatsh. Math. 108, 129-148.

[11] Feichtinger, H. G. and Gröchenig, K. (1992). Nonorthogonal wavelet and Gabor expansions and group representations, in Wavelets and Their Applications, Ruskai, M. B., et al., Eds., Jones and Bartlett, Boston, 353-376.

[12] Feichtinger, H. and Gröbner, P. (1985). Banach spaces of distributions defined by decomposition methods, I, Math. Nachr. 123, 97-120.

[13] Folland, G. B. (1989). Harmonic Analysis in Phase Space, Princeton University Press, Princeton.

[14] Gröchenig, K. (1991). Describing functions: Atomic decomposition versus frames, Monatsh. Math. 112, $1-42$.

[15] Gröchenig, K. (2000). Foundations of Time-Frequency Analysis, Birkhäuser.

[16] Gröchenig, K. and Samarah, S. (2000). Nonlinear approximation with local Fourier bases, Constr. Approx. 16, 317-331.

[17] Stevenson, R. (2003). Adaptive solution of operator equations using wavelet frames, SIAM J. Numer. Anal. 41, 1074-1100.

[18] Torresani, B. (1995). Position-frequency analysis for signals defined on spheres, Signal Process. 43(3), $341-346$. 
Received September 19, 2006

Universität Marburg, Fachbereich Mathematik und Informatik, Hans-Meerwein-Str., Lahnberge 35032 Marburg, Germany

e-mail: dahlke@mathematik.uni-marburg.de

Universität Mannheim, Fakultät für Mathematik, und Informatik, A5

68131 Mannheim, Germany

e-mail: steidl@kiwi.math.uni-mannheim.de

Zuse-Institute-Berlin (ZIB), Takustr. 714195 Berlin, Germany

e-mail: teschke@zib.de 